\title{
SEDIC ABIERTO
}

\section{Últimas actividades}

\section{Celebración de la XII Jornada Internacional de Experiencias Bibliotecarias. Problemaética II}

El 16 de marzo del 2021 se ha celebrado la XII Jornada JIEB (Jornada Internacional de Experiencias Bibliotecarias) bajo el título "PROBLEMÆETICA II".

Las JIEB iniciaron su andadura en el año 2008 y son fruto de la colaboración con el Goethe Institut, la Comunidad de Madrid, la Embajada de EE.UU., SEDIC, Institut Français, Istituto Italiano di Cultura di Madrid e Instituto Internacional en España, entre otros. Las instituciones colaboradoras reúnen a especialistas españoles e internacionales del mundo bibliotecario, con el propósito de analizar y reflexionar sobre las tendencias de la profesión.

En 2021 se ha querido hondar en el principio de igualdad, tratando temas afines a la responsabilidad social en el día a día de nuestra profesión, el trabajo con colectivos desfavorecidos y las relaciones interpersonales, introduciéndonos también en la gestión de conflictos ya sea con colegas o con usuarios y usuarias. El vídeo de la Jornada está en acceso abierto.

\section{Celebración de la XXII Jornada de Gestión de la Información: "Algoritmo \& Compás: EI profesional de la información al son de la inteligencia artificial ¿o no?"}

Tras un parón debido a la pandemia del COVID-19 que no ha permitido la celebración de las Jornadas de Gestión de la Información proyectadas en el año 2020, que se iban a dedicar a la temática de la gestión de la información y de la documentación medioambiental, siendo aplazada a una Jornada futura, finalmente se ha celebrado el día 27 de abril la tan esperada Jornada de Gestión de la Información de SEDIC XXII bajo el título: "Algoritmo \& Compás: El profesional de la información al son de la inteligencia artificial ¿o no?", que ha girado en torno a una temática de actual preocupación en nuestros sector como es la inteligencia artificial y su repercusión en la profesión de gestor de la información y la documentación. Las Jornadas se han desarrollado con un gran éxito de asistencia, y con grandes aportaciones de sus ponentes. Para aquellos que no hayan podido asistir, pero que te tengas interés en escuchar a sus ponentes y mesas, SEDIC ha publicado el vídeo de las Jornadas.

\section{Publicación de un video divulgativo de la profesión bajo el lema: "Rediseñando lo que} somos para renovar lo que hacemos"

El especialista en gestión de la información y de la documentación es un agente fundamental en la sociedad actual. Para llegar a esa sociedad de una forma clara, iniciamos la realización de una 
serie de vídeos divulgativos para promover la labor de los profesionales de la información y trasladarla usando el lenguaje audiovisual como recurso. Os presentamos este primer vídeo que cuenta lo que hacemos y el impacto de nuestro trabajo, destinado a una audiencia amplia.

\section{Celebración de la Jornada Actualízate 2021 “La profesión frente al espejo”}

El día 11 de mayo se ha celebrado la Jornada Actualízate, bajo el título "La profesión frente al espejo". La Jornada Actualízate es una idea innovadora nacida en 2014 que, impulsada por Sociedad Española de Documentación en Información Científica (SEDIC) en colaboración con el Departamento de Biblioteconomía y Documentación de la Universidad Complutense de Madrid, se basa en la organización de una jornada profesional a través de la cual diversas empresas e instituciones, relacionadas con la gestión de la información y/o la gestión documental, comparten sus ideas más innovadores en el desarrollo de su actividad profesional, creando un foro de discusión en torno a la evolución y las tendencias tecnológicas y formativas en el sector de la información documental, buscando así puntos de unión y sinergias entre la actividad profesional y el ámbito universitario (investigador y docente).

Profundizando en esta línea de colaboración empresa-universidad, SEDIC quiere favorecer la innovación y su aplicación práctica en el mundo empresarial convocando, en las mismas jornadas, los Premios al proyecto universitario más innovador en el ámbito de la gestión de la información y la documentación con el objetivo de buscar y destacar públicamente los proyectos teóricos más innovadores que pudieran tener mayor aplicación práctica en el ámbito empresarial entre los trabajos de los estudiantes universitarios de universidades españolas (Trabajo Final de Máster o Trabajo Final de Grado).

\section{Taller gratuito de valoración económica y auditoría interna de los archivos}

Estamos habituados a destacar los valores primarios y secundarios de los documentos de archivos, los primeros son los propios de la fase de archivo administrativo y los segundos son los propios de la fase de archivo histórico, sin embargo, los documentos de archivos, como parte integrante del patrimonio histórico documental, tienen más valores añadidos, uno de esos valores es el económico, ya que son parte del patrimonio mueble de las instituciones.

En este taller se dan las pautas y la metodología para establecer dichos valores en cualquier fondo documental, este valor es importante y se maneja en el mercado obras de arte y antigüedades, sobre todo, de cara a determinar el monto total del valor patrimonial mueble que manejan las instituciones y organizaciones, dicho valor al igual que los valores primarios y secundarios, hacen del archivo un bien tangible más visible dentro de las organización y justifica de una manera más clara de cara a los gestores y directores las inversiones que se realizan en la gestión y conservación de dichas colecciones.

Como complemento a esta formación, se tratará la metodología básica para la realización de la auditoría interna de los archivos. Dicha auditoría tiene como finalidad evaluar la eficacia y eficiencia de la gestión de archivos que repercute en la atención al usuario. Los archivos normalmente son el núcleo de las organizaciones actuales, si bien normalmente se realizan dichas auditorías, desde la primera norma ISO de gestión se ha plasmado la importancia de auditar la gestión del archivo de manera individualizada. Los sistemas de gestión de archivos son tan importantes en las organizaciones que han generado su propia normativa específica para la auditoría de archivos, como es el caso de la norma ISO-UNE 30301. 
Identificadores persistentes: elementos para la interoperabilidad en infraestructuras de investigación

Los identificadores persistentes (PIDs) son elementos centrales en la infraestructura científica, puesto que identifican de forma unívoca todas las entidades que integran el ecosistema de investigación. La identificación persistente de entidades en las infraestructuras de investigación basada en esquemas abiertos facilita la interoperabilidad de los metadatos asociados a dichas entidades y contribuye a la disponibilización de (meta)datos FAIR.

Taller gratuito de retos bibliotecarios: participación ciudadana, tecnologías digitales y diseño creativo.

En estos tiempos de transformación, en los que la digitalización y el uso intensivo de las tecnologías modifican la forma en la que se accede, se produce y se comparte el conocimiento, las bibliotecas se están enfrentando a nuevos y disruptivos desafíos. Uno de esos retos emergentes es cómo afianzar y expandir el rol de las bibliotecas en tanto que agentes comunitarios de transformación social. En este contexto, el taller que os proponemos dará respuesta a la siguiente pregunta: "¿Cómo prototipar proyectos comunitarios desde las bibliotecas a través de las tecnologías digitales?".

\section{Taller gratuito de creación y aplicaciones de las Infografías}

En este taller se muestra la importancia de las infografías en el panorama actual de los contenidos digitales. Se trabajan los distintos tipos de infografías, las aplicaciones y los elementos que pueden formar parte de ellas.

Además, se trabajan algunas de las principales herramientas que se utilizan en la actualidad para la creación de infografías.

Taller gratuito sobre contenido accesible: ¿Qué implica en los pdfs?

Como resultado de la certificación de accesibilidad del Museo y de la obligación de los sitios web públicos de cumplir el RD 1112/2018 es importante conocer las implicaciones en la generación de contenido accesible. El taller está encaminado a dar unas recomendaciones de buenas prácticas para publicar en la web PDFs optimizados y accesibles.

\section{Taller gratuito "Preparación de entrevistas y videoentrevistas"}

En este taller se habla de las entrevistas de trabajo con el objetivo de obtener herramientas que nos faciliten afrontarlas con mayor tranquilidad y seguridad. Entender las características de la situación a la que nos enfrentamos puede ayudarnos a aportar una versión más ajustada al perfil profesional que la empresa está buscando y acercarnos más al logro de nuestro objetivo.

Taller gratuito sobre tipos de manuscritos medievales iluminados: litúrgicos, universitarios, científicos

Cada periodo histórico y artístico se identifica con una tipología de manuscrito determinado. Desde las Biblias Atlánticas del Románico a los Libros de Horas de los siglos XIV y XV, pasando 
por tipos netamente hispanos como los Beatos. Sin olvidar los textos jurídicos o teológicos propios de los estudios universitarios.

Este taller ofrece un breve recorrido por estas categorías proporcionando algunas claves para su descripción y clasificación.

\section{Archivos y Bibliotecas: contenido audiovisual y multimedia en tiempos de pandemia}

Se planteó como un debate sobre el estado de la cuestión audiovisual-multimedia en el ámbito bibliotecario y archivístico en tiempos de pandemia.

Se contó con las opiniones y aportaciones al respecto de los directores Biblioteca Facultad de Traducción y Documentación Universidad Salamanca, Biblioteca Facultad Ciencias de la Información Universidad Complutense, y de la bibliotecaria-documentalista directora Espacio ABiM, presidenta Asociación Bibliotecarios de Misiones (Argentina), profesor de la Universidad la Salle (Colombia) y Soluciones ECM en Evolution Technologies Group.

Estado cuestión generalizada ámbito gestión documental audiovisual en tiempos de pandemia: Alfonso López Yepes, responsable de Archivo audiovisual en Archivoz.

\section{Taller gratuito "El análisis y la optimización del dato"}

Impartido por Jorge Hierro Álvarez, CEO en Agencia Salta Digital, da una visión de la importancia de los datos y la interpretación de la información en las empresas, entidades e instituciones, por medio del empleo de herramientas virtuales y el desarrollo de un Plan de Acción para la consolidación de una estrategia operativa de la estructura organizativa.

\section{Espacio de colaboración IFLA}

Espacio de colaboración IFLA: uno de nuestros los propósitos es fomentar y facilitar que aquellos socios de SEDIC interesados en el trabajo con IFLA, tomen parte y se integren a través de los nuevos entornos de colaboración que, junto con algunos miembros de la junta directiva, gestionarán la representación de SEDIC ante la IFLA. Se trata, por tanto, de crear trabajar en equipo para contribuir a intensificar la presencia de profesionales de nuestro entorno.

\section{SECCIONES EN LAS QUE TENDRÁ PRESENCIA SEDIC:}

19. Preservation and Conservation

21. Information Technology

40. Management of Library Associations

43. Continuing Professional Development and Workplace Learning

49. Environment, Sustainability and Libraries

\section{Espacio de colaboración ICA}

Espacio de colaboración ICA: uno de nuestros los propósitos es fomentar y facilitar que aquellos socios de SEDIC interesados en el trabajo con ICA, tomen parte y se integren a través de los nuevos entornos de colaboración que, junto con algunos miembros de la junta directiva, 
gestionarán la representación de SEDIC ante el ICA. Se trata, por tanto, de crear trabajar en equipo para contribuir a intensificar la presencia de profesionales de nuestro entorno.

\author{
SECCIONES EN LAS QUE TENDRÁ PRESENCIA SEDIC: \\ Sección de Asociaciones Profesionales - SPA \\ Section for Education and Training - SAE
}

\title{
Próximas Actividades
}

\section{Taller gratuito "La comunicación verbal y no verbal en la creación de una estrategia de éxito"}

Gestos, palabras, oratoria, la presencia o saber cómo tratar a cada colectivo, hace que revisemos el esquema de la comunicación y encontremos en las pautas de conducta, una serie de ventajas que permiten ayudar, colaborar y generar el éxito en la interacción del mensaje, entre otros elementos. El taller se impartirá en línea a través de la plataforma ZOOM. (El taller quedará grabado y disponible para socios a través de la pestaña streaming). Se celebrará el viernes 11 de junio de 2021, de 16:00 a 19:00 horas. Hay 50 plazas virtuales disponibles para este taller. Se puede reservar plaza en la intranet mediante el botón en la parte inferior de esta entrada. Las plazas serán confirmadas por mail a los asistentes.

\section{Aplicaciones y servicios para bibliotecas en dispositivos móviles. Curso gratuito y exclusivo para socios de SEDIC. Curso gratuito y exclusivo para socios}

La web móvil y los dispositivos móviles han sido considerados durante varios años una tendencia, hoy son una realidad. Nos encontramos en un momento que recuerda al de los comienzos de Internet, puesto que debemos familiarizarnos con estos dispositivos -que presentan importantes elementos diferenciales con respecto a otras herramientas de información- y comenzar a descubrir posibles utilidades prácticas para nuestras bibliotecas. En este curso se proporcionará al alumno el marco necesario para comprender las implicaciones de los dispositivos móviles para la información y se le dotará de los conocimientos básicos para comenzar a explorar posibles aplicaciones prácticas en bibliotecas, prestando especial atención a soluciones para todos los bolsillos. Se celebrará el 15 de junio de 2021 al 15 de julio del 2021 ,con 300 plazas.

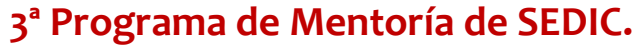

El programa de mentoría de SEDIC es un programa formal de mentoría a través del cual emparejaremos a miembros con más experiencia con aquellos que son nuevos en el sector y que están buscando orientación profesional. El programa es posible gracias al generoso apoyo de los miembros de SEDIC que ofrecen voluntariamente su tiempo como mentores. A cambio de su tiempo, los mentores disfrutan de la oportunidad de contribuir a la comunidad profesional mientras desarrollan sus habilidades de mentoría y coaching.

Se pone en marcha la tercera edición del programa:

Fecha límite para cumplimentar la solicitud: 15 de junio de 2021 
82 Clip de SEDIC, Revista de la Sociedad Española de Documentación e Información Científica

Comienzo del programa: 15 de septiembre de 2021

Finalización del programa: 15 de mayo de 2022 